\title{
Genetic associations of leptin-related polymorphisms with systemic lupus erythematosus
}

Jian Zhao ${ }^{1}$, Hui Wu ${ }^{1}$, Carl D. Langefeld ${ }^{2}$, Kenneth M. Kaufman ${ }^{3,4}$, Jennifer A. Kelly ${ }^{5}$, Sang-

Cheol Bae ${ }^{6}$, Marta E. Alarcón-Riquelme for the BIOLUPUS ${ }^{\natural}$ and GENLES ${ }^{\Uparrow}$ networks ${ }^{5,7}$,

Graciela S. Alarcón ${ }^{8}$, Juan-Manuel Anaya ${ }^{9}$, Lindsey A. Criswell ${ }^{10}$, Barry I. Freedman ${ }^{11}$, Diane L.

Kamen $^{12}$, Gary S. Gilkeson ${ }^{12}$, Chaim O. Jacob ${ }^{13}$, Judith A. James ${ }^{5,14,15}$, Joan T. Merrill ${ }^{16}$, Patrick

M. Gaffney ${ }^{5}$, Kathy Moser Sivils ${ }^{5,14}$, Timothy B. Niewold ${ }^{17}$, Michelle A. Petri ${ }^{18}$, Seung Taek

Song $^{6}$, Hye-jin Jeong ${ }^{6}$, Rosalind Ramsey-Goldman ${ }^{19}$, John D. Reveille ${ }^{20}$, R. Hal Scofield ${ }^{5,15,21}$,

Anne M. Stevens ${ }^{22,23}$, Susan A. Boackle ${ }^{24,25}$, Luis M. Vilá ${ }^{26}$, Deh-Ming Chang ${ }^{27}$, Yeong Wook

Song $^{28}$, Timothy J. Vyse ${ }^{29}$, John B. Harley ${ }^{3,4}$, Elizabeth E. Brown ${ }^{8,30}$, Jeffrey C. Edberg ${ }^{8}$, Robert

P. Kimberly ${ }^{8}$, Bevra H. Hahn ${ }^{1}$, Jennifer M. Grossman ${ }^{1}$, Betty P. Tsao ${ }^{1 *}$ and Antonio La Cava ${ }^{1^{*}}$

${ }^{1}$ Department of Medicine, University of California Los Angeles, Los Angeles, CA; ${ }^{2}$ Department

of Biostatistical Sciences and Center for Public Health Genomics, Wake Forest School of

Medicine, Winston-Salem, NC; ${ }^{3}$ Cincinnati Children’s Hospital Medical Center, Cincinnati, OH;

${ }^{4}$ US Department of Veterans Affairs Medical Center, Cincinnati, OH; ${ }^{5}$ Arthritis and Clinical

Immunology Research Program, Oklahoma Medical Research Foundation, Oklahoma City, OK;

${ }^{6}$ Department of Rheumatology, Hanyang University Hospital for Rheumatic Diseases, Seoul,

Korea; ${ }^{7}$ Pfizer-Universidad de Granada-Junta de Andalucía Center for Genomics and

Oncological Research, Granada, Spain; ${ }^{8}$ Department of Medicine, University of Alabama at

Birmingham, Birmingham, AL; ${ }^{9}$ Center for Autoimmune Diseases Research, Universidad del

Rosario, Bogotá, Colombia; ${ }^{10}$ Rosalind Russell/Ephraim P. Engleman Rheumatology Research

Center, University of California San Francisco, San Francisco, CA; ${ }^{11}$ Department of Internal

Medicine, Wake Forest School of Medicine, Winston-Salem, NC; ${ }^{12}$ Medical University of South 
Carolina, Charleston, SC; ${ }^{13}$ Department of Medicine, University of Southern California, Los Angeles, CA; ${ }^{14}$ Department of Pathology, University of Oklahoma Health Sciences Center, Oklahoma City, OK; ${ }^{15}$ Department of Medicine, University of Oklahoma Health Sciences Center, Oklahoma City, OK; ${ }^{16}$ Clinical Pharmacology, Oklahoma Medical Research Foundation, Oklahoma City, OK; ${ }^{17}$ Department of Immunology, Mayo Clinic, Rochester, MN; ${ }^{18}$ Department of Medicine, Johns Hopkins University School of Medicine, Baltimore, MD; ${ }^{19}$ Northwestern University Feinberg School of Medicine, Chicago, IL; ${ }^{20}$ Rheumatology and Clinical Immunogenetics, University of Texas Health Science Center at Houston, Houston, TX; ${ }^{21}$ US Department of Veterans Affairs Medical Center, Oklahoma City, OK; ${ }^{22}$ Department of Pediatrics, University of Washington, Seattle, WA; ${ }^{23}$ Center for Immunity and Immunotherapies, Seattle Children's Research Institute, Seattle, WA; ${ }^{24}$ University of Colorado School of Medicine, Aurora, CO; ${ }^{25}$ US Department of Veterans Affairs Medical Center, Denver, CO; ${ }^{26}$ Department of Medicine, University of Puerto Rico Medical Sciences Campus, San Juan, Puerto Rico; ${ }^{27}$ National Defense Medical Center, Taipei City, Taiwan; ${ }^{28}$ Seoul National University, Seoul, Korea; ${ }^{29}$ King's College London, London, UK; ${ }^{30}$ Department of Epidemiology, University of Alabama at Birmingham, Birmingham, AL

"The BIOLUPUS network is composed of Johan Frostegård (Huddinge, Sweden), Lennart Truedsson (Lund, Sweden), Enrique de Ramón (Málaga, Spain), José M. Sabio (Granada, Spain), María F. González-Escribano (Sevilla, Spain), Javier Martin (Granada, Spain), Norberto OrtegoCenteno (Granada, Spain), José Luis Callejas (Granada, Spain), Julio Sánchez-Román (Sevilla, Spain), Sandra D’Alfonso (Novara, Italy), Sergio Migliarese (Napoli, Italy), Gian-Domenico Sebastiani (Rome, Italy), Mauro Galeazzi (Siena, Italy), Torsten Witte (Hannover, Germany), 
Bernard R. Lauwerys (Louvain, Belgium), Emoke Endreffy (Szeged, Hungary), László Kovács (Szeged, Hungary), Carlos Vasconcelos (Porto, Portugal) and Berta Martins da Silva (Porto, Portugal). The members of GENLES Network are Hugo R. Scherbarth, Pilar C. Marino, Estela L. Motta, Susana Gamron, Cristina Drenkard, Emilia Menso, Alberto Allievi, Guillermo A. Tate, Jose L. Presas, Simon A. Palatnik, Marcelo Abdala, Mariela Bearzotti, Alejandro Alvarellos, Francisco Caeiro, Ana Bertoli, Sergio Paira, Susana Roverano, Cesar E. Graf, Estela Bertero, Cesar Caprarulo, Griselda Buchanan, Carolina Guillerón, Sebastian Grimaudo, Jorge Manni, Luis J. Catoggio, Enrique R. Soriano, Carlos D. Santos, Cristina Prigione, Fernando A. Ramos, Sandra M. Navarro, Guillermo A. Berbotto, Marisa Jorfen, Elisa J. Romero, Mercedes A. Garcia, Juan C Marcos, Ana I. Marcos, Carlos E. Perandones, Alicia Eimon, Sanatorio Parque and Cristina G. Battagliotti in Argentina; Eduardo Acevedo and Mariano Cucho in Perú; Ignacio García de la Torre, Mario Cardiel Ríos, José Francisco Moctezuma and Marco Maradiaga Ceceña in Mexico.

Corresponding authors: Antonio La Cava or Betty P. Tsao, Division of Rheumatology, David Geffen School of Medicine, University of California Los Angeles, 1000 Veteran Ave. 32-59, Los Angeles, CA 90095-1670. E-mails: alacava@mednet.ucla.edu or btsao@mednet.ucla.edu 


\begin{abstract}
Leptin is abnormally elevated in the plasma of patients with systemic lupus erythematosus (SLE), where it is thought to promote and/or sustain pro-inflammatory responses. Whether this association could reflect an increased genetic susceptibility to develop SLE is not known, and studies of genetic associations with leptin-related polymorphisms in SLE patients have been so far inconclusive. Here we genotyped DNA samples from 15,706 SLE patients and healthy matched controls from four different ancestral groups, to correlate polymorphisms of genes of the leptin pathway to risk for SLE. It was found that although several SNPs showed weak associations, those associations did not remain significant after correction for multiple testing. These data do not support associations between defined leptin-related polymorphisms and increased susceptibility to develop SLE.
\end{abstract}

Keywords: systemic lupus erythematosus, leptin pathway, gene polymorphisms 


\section{Introduction}

The etiopathogenesis of systemic lupus erythematosus (SLE) generally considers an involvement of environmental factors (including epigenetic changes) that could trigger abnormal autoimmune responses, facilitated by sex and hormones, in individuals that carry a predisposing genetic background (1). Therefore, in SLE multiple genetic variants could create favorable conditions for a heightened sensitivity of autoreactive cells to an increased stimulation/activation.

Our group and others have previously shown that leptin is abnormally elevated in SLE patients (2-3). We also showed that leptin in mice could promote SLE autoimmunity (4-5). Whether these findings underlie genetic association(s) between selected leptin polymorphisms and SLE is not known. A recent study suggested an association of a leptin receptor gene polymorphism (LEPRQ223R) with increased susceptibility of SLE in 100 Kashmiri individuals (6). Since analyses on larger numbers of SLE patients and in multiple ethnic groups would better delineate the possibility of association(s) between leptin-related genes and increased risk for SLE, we performed genetic association studies for single nucleotide polymorphisms (SNPs) within multiple leptin-related genes. Criteria for selection were based on the following considerations. Leptin gene ( $L E P$ ) polymorphisms (7) were studied because of their possible roles in abnormal function/catabolism of leptin. For leptin receptor ( $L E P R)$, which exists in six alternatively spliced forms with cytoplasmic domains of different length (8), we assessed polymorphisms of all isoforms (9) because any of them might influence catabolism and/or sustain leptin activity. The polymorphism of PPARG (10-12) was also studied because leptin can downregulate PPAR- $\gamma$ expression for a subsequent increase in the release of pro-inflammatory IL-1 $\beta$, IL-6 and TNF- $\alpha$ (13-14). Finally, the polymorphism of the growth hormone secretagogue receptor GHSR was 
studied because its opposing action on leptin results in the inhibition of the same proinflammatory cytokines (15-16).

Haplotype-tagging SNPs selected from these genes were genotyped by a customized SNP genotyping-array and assessed for association with SLE in 15,706 case-control subjects from four different ancestral groups including European American (EA), African American (AA), East Asian (AS) and Hispanic enriched for the Amerindian-European admixture (HS). 


\section{Materials and Methods}

\section{Subjects' samples collection and SNP genotyping}

To test association of LEPR, PPARG, GHSR and LEP with SLE, we used a large collection of samples from case-control subjects from multiple ethnic groups. These samples were from the collaborative Large Lupus Association Study 2 (LLAS2) and were contributed by participating institutions in the United States, Asia and Europe. All SLE patients met the American College of Rheumatology (ACR) criteria for the classification of SLE (17). LLAS2 samples were processed at the Lupus Genetics Studies Unit of the Oklahoma Medical Research Foundation (OMRF). SNP genotyping was carried out on the Illumina iSelect platform. Subjects with individual genotyping call rate $<0.90$ were removed because of low data quality. Subjects that were duplicated or first degree related were also removed. Both principal component analysis and global ancestry estimation based on 347 ancestry informative markers (AIMs) were used to detect population stratification and admixture, as described in another LLAS2 report (18). After removing genetic outliers, a final dataset of 15,706 unrelated subjects $(8,269$ cases vs. 7,437 controls) was obtained.

According to genetic ancestry, subjects were grouped into four ancestral groups including European American (3,966 cases vs. 3,543 controls), African American (1,527 cases vs. 1,812 controls), East Asian (1,272 cases vs. 1,270 controls) and Hispanic enriched for the AmerindianEuropean admixture (1,504 cases vs. 812 controls).

The study was approved by the Human Subject Institutional Review Boards or the Ethic Committee of each institution. All subjects were enrolled after informed consent had been obtained. 


\section{SNP selection and Statistical analysis}

To avoid the genotyping of all SNPs for the genes of interest yet capture the majority of diversity within each region, we selected haplotype tag SNPs for genotyping according to the Hapmap Project (http://hapmap.ncbi.nlm.nih.gov/cgi-perl/gbrowse/hapmap24_B36/; HapMap public release \#24 of 11/26/2008). In addition, SNPs with potential functional consequences were selected as well for testing. In total, we selected 9 SNPs for $L E P, 17$ SNPs for $L E P R$, 5 SNPs for GHSR and 16 SNPs for PPARG, at an average density of $8.2 \mathrm{~kb}$ per SNP. 32 SNPs that passed data cleaning and quality control measures (7 SNPs for LEP, 10 SNPs for $L E P R$, 3 SNPs for GHSR and 12 SNPs for PPARG, Table 1) were genotyped on the Illumina iSelect platform and subsequently used for genetic association test.

The Hardy-Weinberg equilibrium (HWE) test threshold was set at $P>0.01$ for controls and $P>0.0001$ for cases. SNPs failing the HWE test were excluded from association test. SNPs showing genotyping missing rate $>5 \%$ or showing significantly different genotyping missing rate between cases and controls (missing rate $>2 \%$ and $P_{\text {missing }}<0.05$ ) were excluded from association test. In each ancestral group, SNPs were assessed for association with SLE under a logistic regression model adjusting for gender and the first 3 principal components estimated using AIMs. The trans-ancestry meta-analysis was conducted across all four ancestral groups. For each SNP, if the Cochran's Q statistic showed no evidence of genetic heterogeneity $(P>0.05)$, a fixed effect model was applied. Otherwise, a random effect model was used. All analyses described above were performed using PLINK v1.07 (19). Pairwised LD values shown in Figure 1 were calculated using Haploview 4.2 (20) 


\section{Results}

\section{Genetic association between leptin-related polymorphisms and human SLE}

To test the possibility of common leptin-related variants predisposing to SLE, genetic association studies using htSNPs for the four selected candidate leptin-related genes LEP, LEPR, GHSR and PPARG were performed in different ancestral groups. Information and SNPs scoring are reported in Supplementary Table 1. Using Illumina microarray platform, 15,706 case-control samples from four ethnic groups were genotyped in LLAS2 including European American (EA), African American (AA), East Asian (AS) and Hispanic enriched for the Amerindian-European admixture (HS).

In LEP, the A allele of rs12706832 showed association with decreased risk of SLE in AA (78.3\% in cases vs. $80.6 \%$ in controls, $P=0.0063$, OR=0.84, Table 1$)$. In addition, the A allele of rs3828942 was associated with increased risk of SLE in AA (19.5\% in cases vs. $17.7 \%$ in controls, $P=0.029$, OR=1.16, Table 1)

In LEPR, two SNPs rs6690625 and rs1892535 showed association with decreased risk of SLE in HS (C allele of rs6690625, 25.9\% in cases vs. 28.7\% in controls, $P=0.0011$, OR=0.79; A allele of rs1892535, $25.0 \%$ in cases vs. $26.8 \%$ in controls, $P=0.0068$, OR=0.82, Table 1 ).

In GHSR, the G allele of rs2948694 was associated with decreased risk of SLE in HS (13.7\% in cases vs. $15.3 \%$ in controls, $P=0.019$, $\mathrm{OR}=0.81$, Table 1 ).

In PPARG, two SNPs rs12633551 and rs3856806 were associated with decreased risk of SLE in EA (A allele of rs12633551, 2.8\% in cases vs. 3.3\% in controls, $P=0.022$, $O R=0.80$; A allele of rs3856806, $11.1 \%$ in cases vs. $12.3 \%$ in controls, $P=0.023$, OR=0.88, Table 1 ). 
Although we detected significant association signals at several loci, none of them showed consistent association $(P<0.05)$ in multiple ancestral groups. The meta-analysis combining all four ancestral groups showed that multiple SNPs in PPARG exhibited association with SLE $\left(P_{\text {meta }}=0.031,0.024,0.025,0.0080\right.$ and 0.024 for rs6785890, rs10510410, rs12633551, rs4145574 and rs3856806, respectively, Table 1). After Bonferroni correction for multiple tests, only the association of rs6690625 in $L E P R$ with SLE in HS remained significant ( $P=0.0011$, which was less than the corrected $P=0.05 / 32=0.0016$ ).

Together, these data do not provide evidence that leptin-related genes can increase risk for SLE. 


\section{Discussion}

In SLE, many candidate genes including MHC loci, complement components, mannose-binding protein, Fc- $\gamma$ receptors and pro-inflammatory cytokines have been tested by genetic association studies for risk of SLE (21-22). Recently, the role of leptin receptor polymorphism as a possible contributor to SLE risk was suggested (6). In the LEPR, several SNPs gene have been identified, including the Q223R polymorphism in which an A to G transition in exon 6 (that encodes for the extracellular domain of the leptin receptor) (23) could lead to altered signal transduction by altering binding to leptin and/or by impairing LEPR expression. Afroze and colleagues found that carriers of variant genotype $(A / G+G / G)$ or $G$ allele were at elevated risk for SLE (6). However, the number of SLE patients in that study was so low that it lacked power to reach any relevant conclusion as for the association between leptin receptor and SLE. Conversely, our study uses very large number of SLE subjects and is well powered, thus the conclusions can be considered highly significant.

Genetics plays a key role in the pathogenesis of autoimmune diseases because it can influence the expression and activity of genes that are relevant to the disease. Here we aimed to address whether the abnormally increased levels of leptin in SLE patients could associate to gene polymorphisms, i.e. whether certain leptin-related polymorphisms might contribute to a predisposing SLE background that would sustain hyperleptinemia. Although initial assessments suggested the possibility of associated polymorphisms, extensive analyses on 15,706 individuals of multiple ancestries did not confirm the findings. However, we acknowledge that our results do not exclude the possibility that other polymorphisms in genes different from the ones tested here and related to leptin activities could associate with increased susceptibility to develop SLE. Yet 
such genes should be indirectly related to leptin because both leptin and leptin receptor polymorphisms showed no association with increased SLE risk in this study.

The finding of a lack of polymorphisms association with the leptin pathway reminds the results obtained in genetic association studies of human BLyS and SLE. Like leptin, BLyS is an important pro-inflammatory cytokine that is abnormally elevated in SLE patients (24). However, no polymorphism in BLyS or BlyS receptor BCMA was found associated with SLE $(25,26)$. As for leptin, it might be possible that the increase of BLyS levels in SLE patients could be an indirect consequence of other gene(s) associated with SLE or a consequence of multiple interactions between genes and/or genes and environment. It derives that multivariate analyses on subsets of patients (i.e., based on autoantibody positivity, disease manifestations, organ involvement etc.) should be performed for possible identification of associations and assessment of functional significance. In our case, future studies will need to address whether stratification of SLE patients might identify associations of selected SNPs polymorphisms with defined subsets of patients. 


\section{References}

1. Tsokos, G.C. Systemic lupus erythematosus. N. Engl. J. Med. 365 (2011) 2110-2121.

2. Garcia-Gonzalez A., L. Gonzalez-Lopez, I.C. Valera-Gonzalez, E.G. Cardona-Muñoz, M. Salazar-Paramo, M. González-Ortiz, E. Martínez-Abundis, J.I. Gamez-Nava. Serum leptin levels in women with systemic lupus erythematosus. Rheumatol. Int. 22 (2002) $138-141$.

3. McMahon M., B.J. Skaggs, L. Sahakian, J. Grossman, J. FitzGerald, N. Ragavendra, C. Charles-Schoeman, M. Chernishof, A. Gorn, J.L. Witztum, W.K. Wong, M. Weisman, D.J. Wallace, A. La Cava, B.H. Hahn. High plasma leptin levels confer increased risk of atherosclerosis in women with systemic lupus erythematosus, and are associated with inflammatory oxidised lipids. Ann. Rheum. Dis. 70 (2011) 1619-1624.

4. Amarilyo G., N. Iikuni, F.D. Shi, A. Liu, G. Matarese, A. La Cava. Leptin promotes lupus T-cell autoimmunity. Clin. Immunol. 149 (2013) 530-533.

5. Hahn B.H., E.V. Lourenço, M. McMahon, B. Skaggs, E. Le, M. Anderson, N. Iikuni, C.K. Lai, A. La Cava. Pro-inflammatory high-density lipoproteins and atherosclerosis are induced in lupus-prone mice by a high-fat diet and leptin. Lupus 19 (2010) 913-917.

6. Afroze D., A. Yousuf, R. Ali, F. Kawoosa, T. Akhtar, S. Reshi, Z.A.Shah. Serum leptin levels, leptin receptor gene (LEPR) polymorphism, and the risk of systemic lupus erythematosus in Kashmiri population. Immunol. Invest. 44 (2015) 113-q25.

7. Comuzzie A.G J.E. Hixson, L. Almasy, B.D. Mitchell, M.C. Mahaney, T.D. Dyer, M.P. Stern, J.W. MacCluer, J. Blangero. A major quantitative trait locus determining serum 
leptin levels and fat mass is located on human chromosome 2. Nat. Genet. 15 (1997) 273276.

8. La Cava A., G. Matarese. The weight of leptin in immunity. Nat. Rev. Immunol. 4 (2004) 371-379.

9. Onions K.L., S.C. Hunt, M.P. Rutkowski, C.A. Klanke, Y.R. Su, M. Reif, A.G. Menon. Genetic markers at the leptin (OB) locus are not significantly linked to hypertension in African Americans. Hypertension 31 (1998) 1230-1234.

10. Florez J.C., K.A. Jablonski, M.W. Sun, N. Bayley, S.E. Kahn, H. Shamoon, R.F. Hamman, W.C. Knowler, D.M. Nathan, D. Altshuler, Diabetes Prevention Program Research Group. Effects of the type 2 diabetes-associated PPARG P12A polymorphism on progression to diabetes and response to troglitazone. J. Clin. Endocrinol .Metab. 92 (2007) 1502-1509.

11. Jaziri R., S. Lobbens, R. Aubert, F. Péan, S. Lahmidi, M. Vaxillaire, I. Porchay, N. Bellili, J. Tichet, B. Balkau, P. Froguel, M. Marre, F. Fumeron, DESIR Study Group. The PPARG Pro12Ala polymorphism is associated with a decreased risk of developing hyperglycemia over 6 years and combines with the effect of the APM1 G-11391A single nucleotide polymorphism: the Data From an Epidemiological Study on the Insulin Resistance Syndrome (DESIR) study. Diabetes 55 (2006) 1157-1162.

12. Wei Q., D.R. Jacobs Jr, P.J. Schreiner, D.S. Siscovick, M.W. Steffes, M. Fornage. Patterns of association between PPAR $\gamma$ genetic variation and indices of adiposity and insulin action in African-Americans and whites: the CARDIA Study. J. Mol. Med. 84 (2006) 955-965. 
13. Lappas M., M. Permezel, G.E. Rice. Leptin and adiponectin stimulate the release of proinflammatory cytokines and prostaglandins from human placenta and maternal adipose tissue via nuclear factor- $\kappa B$, peroxisomal proliferator-activated receptor- $\gamma$ and extracellularly regulated kinase 1/2. Endocrinology 146 (2005) 3334-3342.

14. Cabrero A., M. Cubero , G. Llaverías , M. Alegret, R. Sánchez, J.C. Laguna , M. Vázquez-Carrera . Leptin down-regulates peroxisome proliferator-activated receptor $\gamma$ (PPAR- $\gamma$ ) mRNA levels in primary human monocyte-derived macrophages. Mol. Cell. Biochem. 275 (2005) 173-179.

15. Sun Y., P. Wang, H. Zheng, R.G. Smith. Ghrelin stimulation of growth hormone release and appetite is mediated through the growth hormone secretagogue receptor. Proc. Natl. Acad. Sci. USA 101 (2004) 4679-4684.

16. Liu Z.Z., W.G. Wang, Q. Li, M. Tang, J. Li, W.T.Wu, Y.H. Wan, Z.G. Wang, S.S. Bao, J. Fei. Growth hormone secretagogue receptor is important in the development of experimental colitis. Cell. Biosci. 21 (2015) 5:12.

17. Hochberg M.C. Updating the American College of Rheumatology revised criteria for the classification of systemic lupus erythematosus. Arthritis Rheum. 40 (1997) 1725.

18. Sánchez E., A. Rasmussen, L. Riba, E. Acevedo, J.A. Kelly, C.D. Langefeld, I. GarcíaDe La Torre, A.M. Maradiaga-Ceceña, M.H. Cardiel, J.A. Esquivel-Valerio, J. Rodriguez-Amado, J.F. Moctezuma, P. Miranda, C. Perandones, C. Castel, H.A. Laborde, P. Alba, J. Musuruana, A. Goecke, J.M. Anaya, K.K. Kaufman, A. Adler, E.E. Brown, G.S. Alarcón, R.P. Kimberly, J.C. Edberg, L.A. Criswell, G.S. Gilkeson, T.B. 
Niewold, J. Martin, T.J. Vyse, R. Ramsey-Goldman, M. Petri, J.T. Merrill, J.D. Reveille, B.P. Tsao, L. Orozco, V. Baca, J.A. James, J.B. Harley, T. Tusié-Luna, B.A. Pons-Estel, C.O. Jacob, M.E. Alarcón-Riquelme. Impact of genetic ancestry and socio-demographic status on the clinical expression of systemic lupus erythematosus in AmerindianEuropean populations. Arthritis Rheum. 64 (2012) 3687-3694.

19. Purcell S., B. Neale, K. Todd-Brown, L. Thomas, M.A. Ferreira, D. Bender, J. Maller, P. Sklar, P.I. de Bakker, M.J. Daly, P.C. Sham. PLINK: a tool set for whole-genome association and population-based linkage analyses. Am. J. Hum. Genet. 81 (2007) 559575.

20. Barrett J.C., B. Fry, J. Maller, M.J. Daly. Haploview: analysis and visualization of LD and haplotype maps. Bioinformatics 2 (2005) 263-265.

21. Lee Y.H., S.K. Nath. Systemic lupus erythematosus susceptibility loci defined by genome scan meta-analysis. Hum. Genet. 118 (2005) 434-443.

22. Tsuchiya N., A. Kawasaki, B.P. Tsao, T. Komata, G.M. Grossman, K. Tokunaga. Analysis on the association of HLA-DRB1 and TNFA promoter polymorphisms with SLE using transmission disequilibrium test. Genes Immunity 2 (2001) 317-322.

23. Yiannakouris N., M. Yannakoulia, L. Melistas, J.L. Chan, D. Klimis-Zacas, C.S. Mantzoros. The Q223R polymorphism of the leptin receptor gene is significantly associated with obesity and predicts a small percentage of body weight and body composition variability. J. Clin. Endocrinol. Metab. 86 (2001) 4434-4439. 
24. La Cava A. Targeting the BLyS-APRIL signaling pathway in SLE. Clin Immunol. 148 (2013) 322-327.

25. Kawasaki A., N. Tsuchiya, T. Fukazawa, H. Hashimoto, K. Tokunaga. Analysis on the association of human BLYS (BAFF, TNFSF13B) polymorphisms with systemic lupus erythematosus and rheumatoid arthritis. Genes Immun. 3 (2002) 424-429.

26. Kawasaki A., N. Tsuchiya, T. Fukazawa, H. Hashimoto, K. Tokunaga. Presence of four major haplotypes in human BCMA gene: lack of association with systemic lupus erythematosus and rheumatoid arthritis. Genes Immun. 2 (2001) 276-279. 


\section{Acknowledgments}

We thank all subjects for participation in this study, and Erika Magdangal for help with DNA preparation and organization. This work was supported by the US National Institutes of Health grants AR53239 (A.L.C.) AR043814 and AR065626 (B.P.T.), AI083194 (J.B.H., K.M.S., R.P.K., L.A.C., T.J.V., M.E.A.R., C.O.J., B.P.T. and P.M.G.), AR049084 (R.P.K., J.B.H., J.C.E., E.E.B., G.S.A., J.D.R., R.R.G. and M.A.P.), AR064820 (E.E.B., M.A.P., R.R.G., J.D.R. and L.M.V.), TR001417 (J.C.E. and R.P.K.), CA141700 and AR058621 (M.E.A.R.), AR053308 and TR000004 (L.A.C.), AR062755 and RR029882 (G.S.G. and D.L.K.), AR057172 (C.O.J.), GM103510, GM104938, AR053483, AI101934 and AI082714 (J.A.J.), AI063274 (P.M.G.), AR043274 (K.M.S.), AI083790, AI071651 and RR024999 (T.B.N.), AR43727 (M.A.P.), AR002138, AR066464 and TR001422 (R.R.G.), RR023417 (J.D.R.), AR051545 and RR025014 (A.M.S.), AI070304 (S.A.B.), AR042460, AR062277, RR020143 and AI024717 (J.B.H.), AR048311 and AR055385 (E.E.B.), AR033062 (R.P.K.). Additional funds were from the Lupus Foundation of America (B.P.T.), the Alliance for Lupus Research (B.P.T., Y.D., K.M.S., T.B.N., L.A.C., C.O.J. and S.A.B.), the Lupus Research Institute (B.P.T. and T.B.N.), the US Department of Veterans Affairs (Merit Awards; J.B.H. and G.S.G.), the US Department of Defense (PR094002, J.B.H.), the Arthritis National Research Foundation (Eng Tan Scholar Award; J.Z. and T.B.N.), the Arthritis Foundation (A.M.S. and P.M.G.), the Korea Healthcare Technology R\&D Project, Ministry for Health and Welfare, Republic of Korea (HI13C2124 to S.C.B and HI14C1277 to Y.W.S.), the European Science Foundation RNP (BIOLUPUS Research Network), the Wellcome Trust (T.J.V.), Arthritis Research UK (T.J.V.), a Kirkland Scholar Award (L.A.C.), the Wake Forest School of Medicine Center for Public Health 
Genomics (C.D.L.), and UCLA Clinical and Translational Science Institute (CTSI) RR033176 and TR000124. 


\section{Figure legends}

Figure 1. SNPs in leptin-related genes assessed for association with SLE. Genomic

structure, SNP location and pairwise linkage disequilibrium (described as $r^{2}$ ) between SNPs are indicated for A) LEPR, B) PPARG, C) GHSR and D) LEP, respectively. 


\begin{tabular}{|c|c|c|c|c|c|c|c|c|c|c|c|c|c|c|c|c|c|c|c|c|c|}
\hline \multirow{3}{*}{ SNP } & \multirow[b]{3}{*}{ Gene } & \multirow[b]{3}{*}{ Location } & \multirow{3}{*}{$\begin{array}{l}\text { Tested } \\
\text { allele }\end{array}$} & \multicolumn{4}{|c|}{ EA } & \multicolumn{4}{|c|}{ AA } & \multicolumn{4}{|c|}{ AS } & \multicolumn{4}{|c|}{ HS } & \multicolumn{2}{|c|}{ Meta-analysis } \\
\hline & & & & \multicolumn{2}{|c|}{ Frequency } & \multirow[b]{2}{*}{$\boldsymbol{P}$} & \multirow[b]{2}{*}{ OR } & \multicolumn{2}{|c|}{ Frequency } & \multirow[b]{2}{*}{$\boldsymbol{P}$} & \multirow[b]{2}{*}{ OR } & \multicolumn{2}{|c|}{ Frequency } & \multirow[b]{2}{*}{$\boldsymbol{P}$} & \multirow[b]{2}{*}{ OR } & \multicolumn{2}{|c|}{ Frequency } & \multirow[b]{2}{*}{$P$} & \multirow[b]{2}{*}{ OR } & & \\
\hline & & & & SLE & CTRL & & & SLE & CTRL & & & SLE & CTRL & & & SLE & CTRL & & & $\boldsymbol{P}$ & OR \\
\hline rs3806318 & LEPR & chr1:65657945 & $G$ & $27.2 \%$ & $26.8 \%$ & 0.35 & 1.04 & $5.3 \%$ & $5.4 \%$ & 0.91 & 1.01 & $13.8 \%$ & $14.0 \%$ & 0.89 & 0.99 & $17.6 \%$ & $18.4 \%$ & 0.95 & 1.01 & 0.46 & 1.02 \\
\hline 59436740 & LEPR & chr1:65664489 & A & $27.7 \%$ & $28.0 \%$ & 0.84 & 0.99 & $47.9 \%$ & $48.4 \%$ & 0.93 & 1.00 & $18.3 \%$ & $20.2 \%$ & 0.077 & 0.88 & $26.9 \%$ & $26.2 \%$ & 0.29 & 1.08 & 0.65 & 0.99 \\
\hline 2029311 & LEPR & chr1:65755938 & A & $3 \%$ & $0.2 \%$ & 0.33 & 1.45 & $.2 \%$ & $0.3 \%$ & 0.14 & 0.45 & & $21.3 \%$ & 0.41 & 0.94 & $7.7 \%$ & $6.5 \%$ & 0.65 & 1.06 & .62 & .97 \\
\hline 409802 & LEPR & chr1:65 & A & $.4 \%$ & $24.4 \%$ & 0.92 & 1.00 & $.5 \%$ & $18.3 \%$ & 0.54 & 1.04 & & $79.3 \%$ & 0.70 & 0.97 & $24.1 \%$ & & 0.22 & 0.92 & 0.65 & .99 \\
\hline 370791 & LEPR & chr1:65824816 & G & $25.7 \%$ & $25.4 \%$ & $0.69 *$ & 1.02 & $29.6 \%$ & $28.6 \%$ & 0.31 & 1.06 & $80.7 \%$ & $80.2 \%$ & 0.63 & 1.04 & $26.8 \%$ & $27.2 \%$ & $0.68^{*}$ & 0.97 & 0.42 & 1.02 \\
\hline 137101 & LEPR & chr1:65 & G & $42.8 \%$ & $43.0 \%$ & 0.69 & 0.99 & $56.0 \%$ & $54.7 \%$ & 0.13 & 1.08 & $86.8 \%$ & $86.8 \%$ & 0.98 & 1.00 & $41.9 \%$ & $45.3 \%$ & $0.012 *$ & 0.85 & 0.59 & 0.99 \\
\hline 90422 & LEPR & chr1:65 & A & $36.7 \%$ & $37.0 \%$ & 0.74 & 0.99 & $56.5 \%$ & $55.5 \%$ & 0.22 & 1.07 & $87.8 \%$ & $87.0 \%$ & 0.42 & 1.07 & $41.0 \%$ & $43.3 \%$ & 0.037 & 0.88 & 0.81 & 0.99 \\
\hline 3179183 & LEPR & chr1:658 & c & $.8 \%$ & $18.0 \%$ & 0.11 & 1.07 & $20.3 \%$ & $18.5 \%$ & 0.23 & 1.08 & $7.4 \%$ & $7.7 \%$ & 0.63 & 0.95 & $18.0 \%$ & $17.2 \%$ & 0.54 & 1.05 & 0.067 & 1.06 \\
\hline 625 & LEPR & chr1:658 & c & $.7 \%$ & $17.8 \%$ & 0.82 & 0.99 & $3.2 \%$ & $31.5 \%$ & $0.097 *$ & 1.10 & $78.6 \%$ & $78.6 \%$ & 0.98 & 1.00 & $25.9 \%$ & $28.7 \%$ & 0.0011 & 0.79 & 0.54 & 0.98 \\
\hline 1892535 & LEPR & chr1: & A & $.0 \%$ & $17.3 \%$ & 0.70 & 0.98 & $1.9 \%$ & $14.6 \%$ & 0.61 & 1.04 & $76.5 \%$ & $76.0 \%$ & 0.72 & 1.02 & $25.0 \%$ & $26.8 \%$ & 0.0068 & 0.82 & 0.31 & 0.97 \\
\hline & PPARG & chr3: & $\mathrm{A}$ & $24.0 \%$ & $24.7 \%$ & 0.26 & 0.96 & $8.0 \%$ & $28.3 \%$ & 0.88 & 1.01 & $49.8 \%$ & & 0.090 & 0.91 & $37.3 \%$ & $37.0 \%$ & $0.076^{*}$ & 0.89 & 0.031 & 0.94 \\
\hline 36188 & PPARG & chr3:12 & G & $2.6 \%$ & $3.0 \%$ & 0.11 & 0.84 & $3.4 \%$ & $3.4 \%$ & 0.47 & 1.11 & $27.6 \%$ & $29.7 \%$ & 0.14 & 0.91 & $1.7 \%$ & $2.2 \%$ & 0.41 & 0.83 & 0.0655 & 0.91 \\
\hline 10510410 & PPARG & chr3:12321738 & c & $23.6 \%$ & $24.4 \%$ & 0.26 & 0.96 & $29.7 \%$ & $30.6 \%$ & 0.52 & 0.96 & $29.1 \%$ & $31.5 \%$ & 0.07 & 0.90 & $35.6 \%$ & $34.8 \%$ & 0.28 & 0.93 & 0.024 & 0.94 \\
\hline 12633551 & PPARG & chr3:12335494 & A & $2.8 \%$ & $3.3 \%$ & 0.022 & 0.80 & $0.7 \%$ & $0.4 \%$ & 0.071 & 1.93 & $24.4 \%$ & $26.1 \%$ & 0.19 & 0.92 & $1.4 \%$ & $2.0 \%$ & 0.28 & 0.77 & 0.025 & 0.89 \\
\hline 4145574 & PPARG & chr3:12 & G & $22.2 \%$ & $23.1 \%$ & 0.13 & 0.94 & $1.1 \%$ & $21.1 \%$ & 0.99 & 1.00 & $28.1 \%$ & $30.5 \%$ & 0.077 & 0.89 & $32.6 \%$ & $33.6 \%$ & $0.031 *$ & 0.86 & 0.0080 & 0.93 \\
\hline & PPARG & chr3: & A & & & 0.61 & 1.02 & $.6 \%$ & $21.4 \%$ & & 0.96 & & & 0.97 & 1.00 & $27.6 \%$ & & & 1.00 & 0.90 & 1.00 \\
\hline & PPARG & chr3: & G & $\%$ & $19.2 \%$ & 0.18 & 1.06 & $6 \%$ & $5.7 \%$ & 0.73 & 0.96 & $46.9 \%$ & $47.6 \%$ & 0.59 & 0.97 & $24.5 \%$ & $22.2 \%$ & 0.21 & 1.10 & 0.30 & 1.03 \\
\hline 709151 & PPARG & chr3:12 & A & $35.1 \%$ & $34.6 \%$ & 0.72 & 1.01 & $13.3 \%$ & $13.5 \%$ & 0.68 & 1.03 & $42.7 \%$ & $43.3 \%$ & 0.71 & 0.98 & $22.7 \%$ & $24.4 \%$ & 0.69 & 0.97 & 0.93 & 1.00 \\
\hline 51175543 & PPARG & chr3:12441433 & G & $35.2 \%$ & $35.1 \%$ & 0.93 & 1.00 & $3.4 \%$ & $13.7 \%$ & 0.71 & 1.03 & $43.2 \%$ & $43.3 \%$ & 1.00 & 1.00 & $23.0 \%$ & $24.5 \%$ & 0.77 & 0.98 & 0.97 & 1.00 \\
\hline 5544 & PPARG & ch & A & $33.1 \%$ & $32.4 \%$ & 0.32 & 1.04 & $0.3 \%$ & $10.7 \%$ & 0.86 & 0.98 & $40.2 \%$ & $40.9 \%$ & 0.60 & 0.97 & $21.5 \%$ & $22.8 \%$ & 0.89 & 0.99 & 0.71 & 1.01 \\
\hline & PPARG & chr3 & c & & $36.9 \%$ & 0.97 & 1.00 & $4.3 \%$ & $13.9 \%$ & $0.29 *$ & 1.08 & $43.6 \%$ & $45.0 \%$ & 0.35 & 0.95 & $24.8 \%$ & $26.3 \%$ & 91 & 0.99 & 0.92 & 1.00 \\
\hline & PPARG & & & & & 0.023 & 0.88 & $5 \%$ & 5.8 & & 1.02 & & & 0.78 & 0.98 & $9.9 \%$ & & & 0.84 & 0.024 & \\
\hline & GHSR & 143 & $\mathrm{~A}$ & $.0 \%$ & $29.5 \%$ & 0.27 & 0.96 & $8.9 \%$ & $8.7 \%$ & 0.8 & 1.02 & $45.5 \%$ & $47.3 \%$ & 0.25 & 0.94 & $33.3 \%$ & $30.2 \%$ & 0.20 & 1.09 & 0.45 & 0.98 \\
\hline 52948694 & GHSR & chr3:173647857 & G & $10.4 \%$ & $10.3 \%$ & 0.83 & 1.01 & $10.1 \%$ & $10.6 \%$ & 0.65 & 0.96 & $35.0 \%$ & $33.8 \%$ & 0.44 & 1.05 & $13.7 \%$ & $15.3 \%$ & 0.019 & 0.81 & 0.61 & 0.98 \\
\hline rs572169 & GHSR & chr3:173648421 & A & $28.8 \%$ & $29.6 \%$ & 0.17 & 0.95 & $8.9 \%$ & $8.9 \%$ & 0.89 & 0.99 & $45.8 \%$ & $47.8 \%$ & 0.19 & 0.93 & $33.0 \%$ & $30.2 \%$ & 0.28 & 1.08 & 0.22 & 0.97 \\
\hline 2278815 & LEP & chr7:127 & $\mathrm{G}$ & $44.7 \%$ & $44.6 \%$ & 0.97 & 1.00 & $5.3 \%$ & $86.4 \%$ & 0.17 & 0.90 & $24.8 \%$ & $23.1 \%$ & 0.16 & 1.10 & $51.7 \%$ & $49.7 \%$ & 0.59 & 1.04 & 0.74 & 1.01 \\
\hline & LEP & & A & & & 0.77 & 1.01 & $.3 \%$ & $80.6 \%$ & 0.0063 & 0.84 & 25 & $23.7 \%$ & 0.15 & 1.10 & $50.9 \%$ & & 98 & 1.00 & 78 & \\
\hline & LEP & & 1 & & & 0.92 & 1.00 & $.5 \%$ & 46. & & 1.06 & & & 0.16 & 0.91 & $47.5 \%$ & & & 1.01 & 92 & \\
\hline 0956 & LEP & 8323 & A & $36.6 \%$ & $37.0 \%$ & 0.70 & 0.99 & $3.6 \%$ & $16.7 \%$ & 0.14 & 1.10 & $21.2 \%$ & $20.9 \%$ & 0.78 & 1.02 & $42.2 \%$ & $38.8 \%$ & 0.16 & 1.09 & 0.33 & 1.03 \\
\hline & LEP & chr7:127678676 & A & $36.6 \%$ & $37.1 \%$ & 0.72 & 0.99 & $18.4 \%$ & $16.7 \%$ & 0.16 & 1.10 & $21.2 \%$ & $20.8 \%$ & 0.74 & 1.02 & $42.3 \%$ & $38.8 \%$ & 0.16 & 1.10 & 0.32 & 1.03 \\
\hline 3828942 & LEP & chr7:127681541 & A & $43.2 \%$ & $43.5 \%$ & 0.64 & 0.98 & $19.5 \%$ & $17.7 \%$ & 0.029 & 1.16 & 74.4\% & $76.4 \%$ & 0.10 & 0.90 & $42.2 \%$ & $41.6 \%$ & 0.41 & 1.05 & 0.86 & 1.00 \\
\hline s11761556 & LEP & chr7:127684305 & C & $45.3 \%$ & $45.4 \%$ & 0.91 & 1.00 & | $82.2 \%$ & $83.0 \%$ & 0.38 & 0.94 & | <0.5\% & $24.6 \%$ & 0.12 & & $51.7 \%$ & $50.5 \%$ & 0.98 & 1.00 & 0.70 & 1.01 \\
\hline
\end{tabular}

Position of each SNP is based on NCBI36/hg18. Significant association signals $(P<0.05)$ are highlighted in bold. For SNPs not passing our criteria of quality control, the $P$ value is annotated with "*". 
A

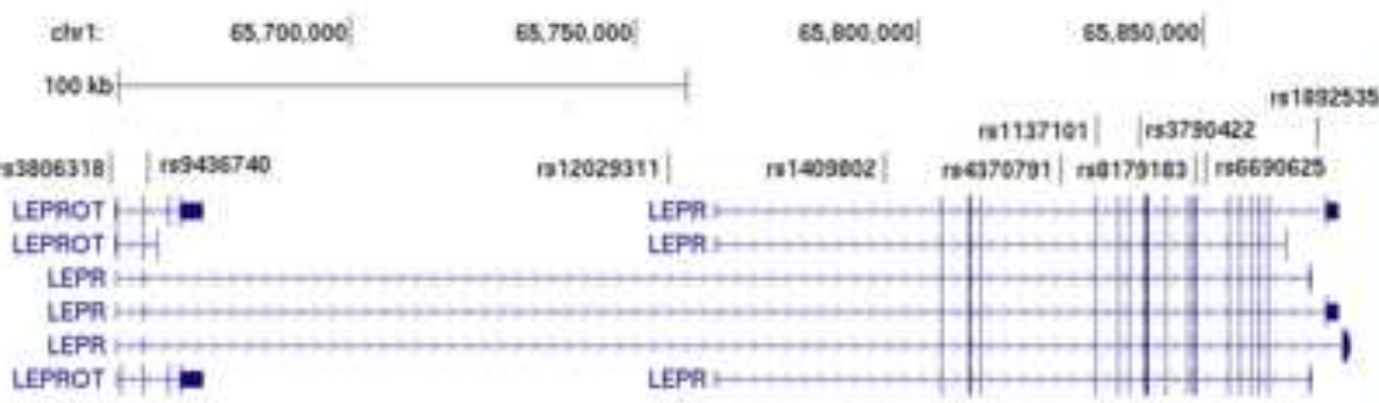

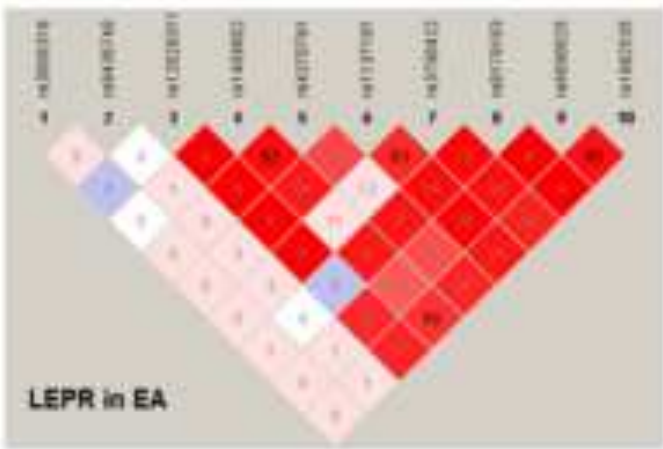

B

cher: 12,300000

$12,250,080$

$12,400,060$

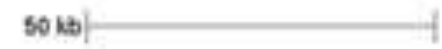

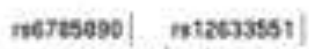

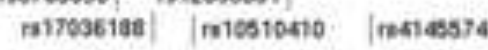

in117sse4

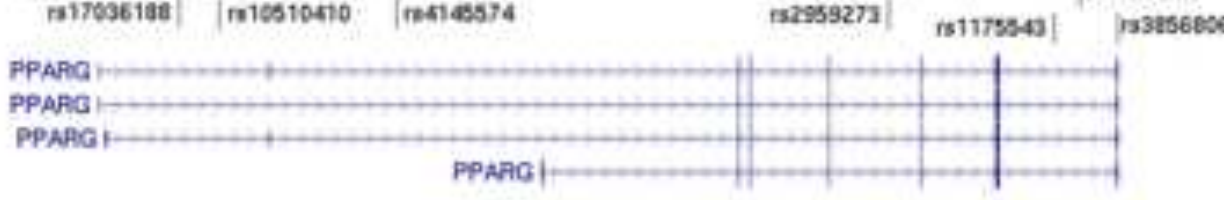

PPARG in EA

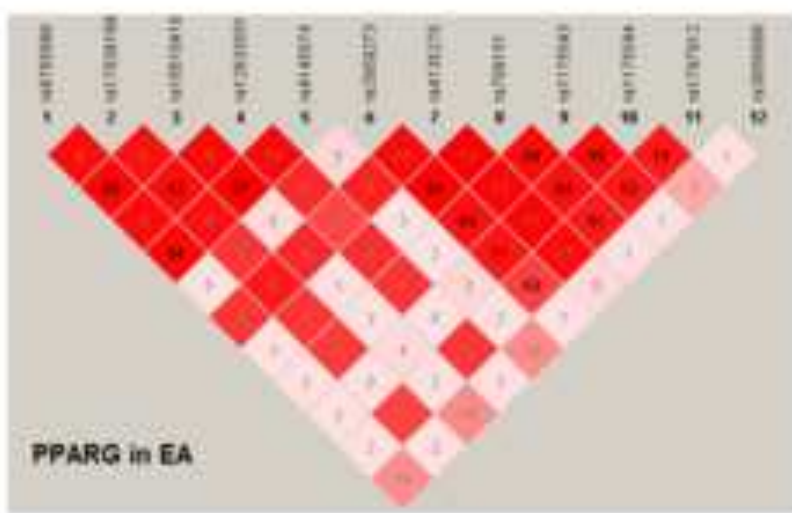

C
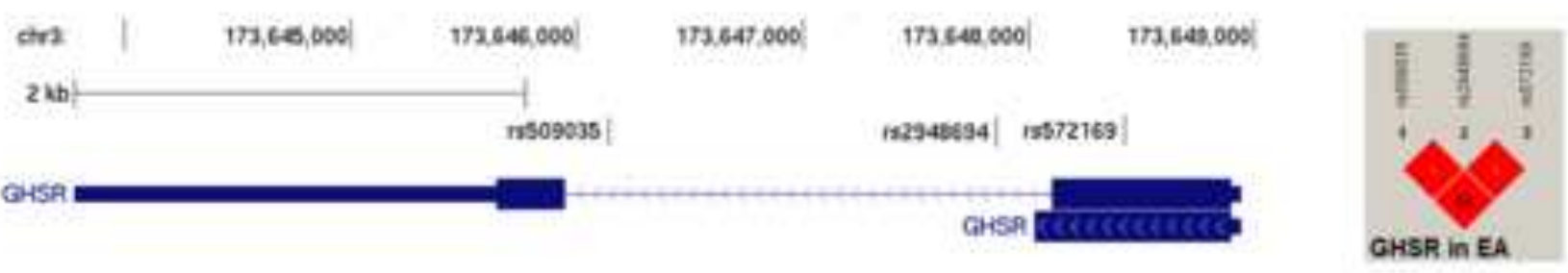

D

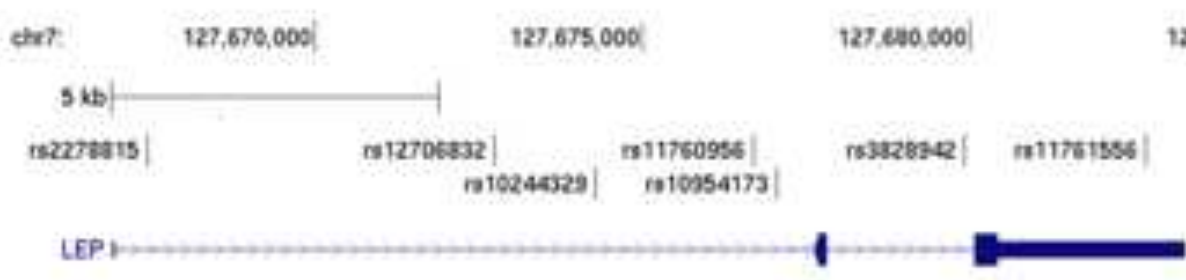

$127,6 a s, 000)$

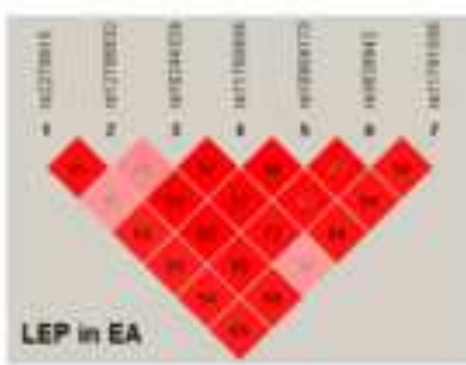

